\title{
Secular Evolution in the Milky Way
}

\section{Victor Debattista}

Jeremiah Horrocks Institute, University of Central Lancashire, Preston, Lancashire, PR1 2HE, United Kingdom, email: vpdebattista@gmail.com

\begin{abstract}
I will review the secular evolution of the Milky Way disk and bulge with particular attention to the bulge and disk. Evidence for the importance of stellar migration in the Solar neighbourhood will be presented. The upcoming Gaia satellite will lead to a wealth of new data with which to explore these effects.
\end{abstract}

